Expert Rev Neurother. 2009 October ; 9(10): . doi:10.1002/pmic.200800802.

\title{
Radiation-induced gliomas
}

\author{
Gautam Prasad and Daphne A. Haas-Kogan ${ }^{1}$
}

\begin{abstract}
Radiation-induced gliomas represent a relatively rare but well-characterized entity in the neurooncologic literature. Extensive retrospective cohort data in pediatric populations after therapeutic intracranial radiation show a clearly increased risk in glioma incidence that is both patient ageand radiation dose/volume-dependent. Data in adults are more limited but show heightened risk in certain groups exposed to radiation. In both populations, there is no evidence linking increased risk associated with routine exposure to diagnostic radiation. At the molecular level, recent studies have found distinct genetic differences between radiation-induced gliomas and their spontaneously-occurring counterparts. Clinically, there is understandable reluctance on the part of clinicians to re-treat patients due to concern for cumulative neurotoxicity. However, available data suggest that aggressive intervention can lead to improved outcomes in patients with radiationinduced gliomas.
\end{abstract}

\section{Keywords}

glioblastoma; radiation; secondary malignancy; gliomas; pediatric neuro-oncology

\section{Glioblastoma}

Glioblastomas (GBMs) are the second most common primary neoplasm of the central nervous system (CNS) in adults with an incidence in the United States of approximately 17,400 new cases (17.6\% of all CNS malignancies) reported in 2004-2005 [101]. During the same time period, incidence of GBMs in children ages 0-14 was approximately $120(2.5 \%$ of all CNS malignancies) and for children ages 15-19 it was approximately 80 (7.2\% of all CNS malignancies). In adults, the publication of the landmark trial by Stupp et al. has changed the standard of care for treatment of GBMs; current standard-of-care for adult GBMs is post-operative concurrent temozolomide and radiation followed by adjuvant temozolomide [1]. This has resulted in a median survival of 14.6 months and is the benchmark for current and future clinical trials. In the pediatric population the standard of care for high-grade gliomas is currently evolving but many clinicians have extrapolated treatment from adult data for use in children. The Children's Oncology Group recently completed a Phase II study (ACNS0126) in pediatric patients with high-grade glioma using concurrent temozlomide and radiation followed by concurrent temozolomide [102]. Eventfree survival (EFS) for 99 eligible high-grade glioma patients on ACNS0126 was compared to a similar cohort of 122 patients from CCG-945, which was open to accrual between 1985 and 1992. Outcome for ACNS0126 did not significantly differ from historical controls. For ACNS0126, one-year EFS was $39 \pm 5 \%$ and one-year overall survival (OS) was $71 \pm 5 \%$.

\footnotetext{
${ }^{1}$ Author for correspondence University of California San Francisco, Helen Diller Family Cancer Center, Department of Radiation Oncology, 1600 Divisadero Street, Suite H1031, San Francisco CA 94143-1708, Tel: +1 415353 7187, Fax: +1 4153539883.
} 


\section{Definition of radiation-induced glioblastoma}

The terms glioblastoma (GBM), glioblastoma multiforme, glioma, and malignant astrocytoma are often used interchangeably in the literature. To be precise, this review defines "GBM" as a World Health Organization (WHO) grade IV astrocytoma whereas the term "high-grade glioma" refers to both WHO grade IV astrocytomas and WHO grade III astrocytomas (also known as anaplastic astrocytomas). Since Grade III astrocytomas are relatively rare compared to Grade IV, the two are frequently combined in clinical trials. When cited studies do not adhere to this precise definition, their criteria will be stated.

Radiation-induced malignancies were defined in a classic study by Cahan et al. [13] examining sarcoma development in irradiated bone. Briefly, they defined four criteria for radiation-induced malignancies: (1) presence of tumor in a previously irradiated region, (2) sufficient latency time between the original and new tumors, generally measured in years, (3) histology of the new tumor must be distinct from the original, and (4) no history of disease predisposing to tumor development (e.g. Li Fraumeni's disease).

\section{Epidemiology of radiation-induced gliomas [pediatric]}

\section{Pediatric cohorts followed after therapeutic radiation}

Historically, pediatric populations have been recognized as more radiosensitive than adults. Therefore, there have been a number of studies evaluating development of intracranial tumors in pediatric populations after treatment with ionizing radiation. The Childhood Cancer Survivor Study consists of a cohort of 14,361 five-year childhood cancer survivors [4]. This cohort included patients under the age of 21 treated for a wide variety of cancers at one of 26 collaborating institutions. An analysis of these patients showed that 40 went on to develop gliomas (including gliosarcomas, oligodendrogliomas, and juvenile pilocytic astrocytomas) — an overall 8.7 standardized incidence ratio (SIR) compared to matched controls. Ninety percent of these 40 patients were originally treated for leukemia, CNS tumor, or lymphoma and $90 \%$ received some form of radiation. The ERR (excess relative risk)/Gy was linear with a slope of 0.33. Subset analyses showed greatest SIR for children younger than 5 years of age at the time their original diagnosis $(\operatorname{SIR}=14.5)$ and for those in the first 15 years of follow-up (years 5-9, SIR $=13.9$; years 10-14, SIR $=11.2$; years 15-19, SIR 3.04; years $\geq 20$, SIR 1.28). After adjusting for radiation, neither the original cancer nor chemotherapy was associated with increased risk.

In addition to treatment for malignant diseases, pediatric patients were also treated with ionizing radiation for benign conditions. A retrospective analysis of 10,834 pediatric patients treated with low-dose radiation (median estimated brain dose of $1.5 \mathrm{~Gy}$ ) for tinea capitis resulted in 21 cases of malignant tumors (defined as gliomas of all types, including ependymomas) [5]. This represented an ERR/Gy of 1.98 compared to matched controls. The ERR/Gy decreased with increasing age from 3.56 at age $<5$ years to 0.47 at age $\geq 10$ years $(P=0.037)$. A pooled Swedish database of 28,008 infants $(<18$ months of age) treated for scalp hemangiomas with a much lower mean absorbed intracranial dose $(0.07 \mathrm{~Gy})$ also revealed an increased risk of CNS tumor development [6]. Specifically they found a SIR of 1.42 with an ERR of 2.7/Gy. Though the study did not statistically separate gliomas (41\% of all tumors) from other types of CNS cancers, there was a strong association between increasing dose and CNS tumor development $(P=0.005)$. Decreasing age $(P=0.017)$ was also an important predictor of CNS tumors.

\section{Are radiation-induced gliomas an issue with modern radiotherapy?}

Given the long latency period between irradiation and development of secondary malignancies, many studies have necessarily focused on older cohorts of pediatric patients. 
However, if many of these same patients were treated with modern linear accelerators that are able to deliver more conformal radiation doses, would the risk of radiation-induced gliomas remain elevated? A recent retrospective study of 4,581 pediatric cancer patients in France treated with radiation indicates that many secondary malignancies actually occur in lower dose areas [25]. Specifically, $31 \%$ of secondary malignancies (14\% in the CNS) occurred in volumes that received < $2.5 \mathrm{~Gy}$. Interestingly, in $6 \%$ of patients' (43\% in the CNS) secondary malignancies occurred in volumes that received $<0.1 \mathrm{~Gy}$. Modeling peripheral dose in pediatric patients has been problematic because much of the data were extrapolated from published literature using adult phantoms. However, more recent data using pediatric phantoms suggest that while doses may fall off rapidly near the target, peripheral doses remain relatively high with intensity modulated radiation therapy compared to $3 \mathrm{D}$ conformal radiation [26, 27].

\section{Pediatric cohort followed after diagnostic imaging}

Given the general sensitivity of pediatric populations to radiation, there has been understandable concern in the medical community about the risk of secondary malignancies after repeat exposure to diagnostic x-rays. A recent review by Linet et al. summarized results of six separate studies of brain tumor development after diagnostic x-ray exposure to the skull, head/neck, chest, or teeth [14]. In general, there did not appear to be a demonstrably increased risk of malignancy after radiation with two exceptions. In one case there was a statistically significant ERR of 6.7 in children with a history of $\geq 5$ full-mouth dental radiographs with a latency of at least 10 years. A second study found a dose-response for chest and skull x-rays with a 5-year latency, though, in retrospect, this may have been confounded by imaging as a result of early symptoms of intracranial neoplasms.

\section{Individual case studies after therapeutic radiation}

Two recent publications $[3,10]$ summarized results of over 100 individual case studies of radiation-induced gliomas in the literature. Common conclusions reached across these diverse cases include: (1) radiation volume was more important than total dose in radiationinduced glioblastoma (RIG) development, (2) the majority of patients ( 60\%) with RIGs were young adults and children, (3) prophylactic cranial irradiation for leukemia was statistically the most common etiology, and (4) mean latency time was on the order of several years (range: 9-17).

\section{Summary}

Data for pediatric secondary malignancies after therapeutic or diagnostic radiation are summarized in Table 1. After intracranial radiation with therapeutic intent (dose range: 0.02 Gy $-45+$ Gy) it is clear, based on several very large pediatric cohorts, that there is an elevated risk of brain tumors in general and gliomas specifically. Furthermore, the risk of secondary malignancy is increased at younger ages $(<5$ years $)$ and at higher treatment volumes and doses. There does not appear to be a dose threshold for secondary glioma development--a potential problem in modern linear accelerators that often deposit low doses far removed from the target. In terms of diagnostic radiation, predominantly administered through low-dose skull radiographs, the majority of studies fail to identify a statistically significant link with subsequent development of intracranial tumors.

\section{Epidemiology of radiation-induced gliomas [adult]}

\section{Adult cohort of atomic bomb survivors}

A life span study of atomic bomb survivors in Hiroshima and Nagasaki, Japan followed a cohort of 80,160 individuals [7]. These registries were first created in 1957 and 1958, respectively, or 12 and 13 years after radiation exposure. Meningiomas were most 
commonly found, followed by gliomas and other neuroepithelial tumors, with a total of 44 recorded cases. Specifically, 13 cases of glioblastoma and 11 of anaplastic astrocytoma occurred. Interestingly, despite the malignant nature of gliomas in general, $23 \%$ of cases were discovered at autopsy while remaining cases were diagnosed after clinical symptoms of motor deficits, seizures, or headaches. For men the adjusted incidence rate (IR) was 3.5 while it was 2.0 for women. When all cases (clinically silent and symptomatic) were taken in aggregate, there was an increasing incidence with increasing age $(P=0.006)$. This trend may represent an imaging bias as older individuals in the cohort would have been much more likely to have modern CT/MRI-based imaging to aid in their diagnosis.

\section{Adult cohort of nuclear industry workers}

The largest ever retrospective cohort of radiation workers in the nuclear industry included more than 400,000 workers in 15 countries [10]. One of the advantages of this study was the accuracy of quantifying radiation exposure through dosimetry badges. Overall there was an increase in radiation-related cause-specific mortality that corresponded to an ERR of 0.42/ $\mathrm{Sv}(P=0.02)$. A relative risk of 1.04 at $0.1 \mathrm{~Sv}$ was calculated. With the exception of lung cancer, no individual cancer type (including CNS malignancies) showed significantly elevated ERRs. In addition there were no statistically significant effects based on age of exposure.

Cleanup workers after the nuclear disaster in Chernobyl were also retrospectively examined as a cohort for cancer risk [11]. Though their average radiation dose was relatively low at $0.1 \mathrm{~Gy}$, it is clear that they received higher exposure than the average nuclear plant worker. Two cohorts ( 10,000 individuals) were combined from the countries of Latvia and Estonia and had a combined SIR for all cancers of 1.53 with a statistically significant elevation in brain cancers (SIR 2.14). However there did not appear to be evidence for a dose response and the data may be skewed by increased surveillance.

\section{Adult cohort followed after repeated CT scans}

Though CT scans represent only $15 \%$ of total imaging in the US, they represent about half of the exposure to radiation. Therefore, it has been postulated by many that repeat CT scans cause elevated cancer risks. Based on results a recent large retrospective cohort of $\sim 30,000$ individuals in a major metropolitan hospital, one-third of patients had five or more CT scans and 5\% had more than $20 \mathrm{CT}$ scans [12]. Based on assumptions of Biological Effects of Ionizing Radiation (BEIR) VII methodology, the lifetime attributable risk (LAR) is $0.7 \%$. In other words, the baseline cancer rate in this cohort would be increased $0.7 \%$ over baseline. The top percentile of patients (those who were imaged most frequently) had a 2.7\% LAR. This study is complicated by the fact that up to $60 \%$ of patients with high LAR were being imaged for active malignancies. Since the study is theoretical, no risks for specific types of tumors are speculated upon.

\section{Adults treated with therapeutic radiation}

For adult patients, many radiation oncologists quote a rough estimate of secondary malignancy of $1-2 \%$ per decade after therapeutic radiation. These figures have been extrapolated from long-term follow-up of patients treated for prostate [30] and cervical cancer [31] where surgical controls can be compared to their radiation counterparts. Though the magnitude of risk is far less for adults than children, it remains statistically significant and is more pronounced in long-term survivors. For CNS tumors, radiation is often the standard of care or remains an important salvage treatment. Thus, it is very difficult to have matched cohorts (as for early-stage prostate and cervical cancer) comparing irradiated and non-irradiated adults. 


\section{Spontaneously-occurring versus radiation-induced gliomas}

\section{Molecular genetic alterations in radiation-induced gliomas}

Traditionally, GBMs have been sub-divided into primary and secondary tumors. The former constitutes the vast majority (> 90\%) of GBMs and consists of tumors that arise de novo. Secondary GBMs ( 5\%) arise from lower grade astrocytomas. Each category, while heterogeneous, has been associated with distinct genetic changes [2]. Differences between genetic alterations in radiation-induced gliomas and those in their spontaneously occurring counterparts are particularly relevant and may shed light on their respective molecular pathogeneses. A pathologic study of nine cases of gliomas arising 5 to 23 years after exposure to therapeutic intracranial radiation examined expression of six major genes [8]. PCR and sequencing of p53, PTEN, K-ras, EGFR, p16, and MTAPdid not demonstrate any major deviations from spontaneously occurring gliomas. However, radiation-induced gliomas were of higher grades, occurred at younger ages ( 6 of 9 patients presented at age < 30 years old), and presented at atypical sites (e.g. suprasellar region, cerebellum).

A more recent study evaluated five RIG specimens using microarray analysis [9]. RIGs were found to be genetically more homogenous than pediatric spontaneously occurring glioblastomas with 2-fold higher number of conserved genes. Venn diagram analysis showed RIGs to have 39\% overlap in their 100 highest overexpressed genes with pilocytic astrocytomas, while only showing $2 \%$ overlap with pediatric GBMs. Three genes in particular were highly over-expressed, including ErB3 and Sox10 (both of which were previously implicated in astrocyte development) and PDGFa. These findings were confirmed by Western blot. Although not specifically validated in RIGs, preclinical data suggest that these three targets can be used in glioma therapy. Inhibition of ErbB3 through disruption of post-translational processing increased radiosensitivity of glioma cells [21]. PDGFa conjugated with I-125 radio-isotopes has been shown to preferentially accumulate in glioma cells [22]. Finally, Sox10 was independently found to act synergistically with PDGFB to promote glioma development [23].

\section{Clinical differences in radiation-induced gliomas}

Radiation-induced gliomas do not necessarily represent a distinct clinical entity. The median survival of patients with RIGs is approximately 11 months with one-, 2-, and 5-year overall survival rates of $37 \%, 13 \%$, and $4 \%$, respectively [3]. Thess survival rates are comparable or slightly less than patients who present with spontaneously occurring gliomas.

\section{Treatment of radiation-induced gliomas}

Since RIGs, by definition, occur within a previously irradiated field, there is understandable reluctance on the part of clinicians to aggressively treat them. Nevertheless, a retrospective analysis by Paulino et al.[3] showed decidedly inferior outcomes in patients who did not receive re-irradiation for RIGs. Of 85 patients evaluated, 35 underwent re-irradiation with a mean dose of $50 \mathrm{~Gy}$ (range: $30-76 \mathrm{~Gy}$ ) with reported 2-year survival of $20.5 \%$. In contrast, the 50 patients who were not irradiated had a 2 -year survival of $3.0 \%(P=0.0009$, log-rank test).

\section{Tolerance of normal brain to radiation}

Classically, dose limits for partial brain and whole brain radiation have been set at $60 \mathrm{~Gy}$ and $50 \mathrm{~Gy}$, respectively [28]. More recent data, however, has challenged this view of radiation tolerance for normal human brain. Using the linear quadratic formula, a mathematical construct used to describe cell survival as a function of total dose and fraction size, Mayer and Sminia found that re-irradiated normal brain tissue tolerated acumulative 
normalized total dose ( $\left.\mathrm{NTD}_{\text {cumulative }}\right)$ of $>100 \mathrm{~Gy}$ at conventional fractionation [29]. This was predicated on assumptions that normal brain was classified as a late-responding tissue $(\alpha / \beta=2 \mathrm{~Gy})$ and that fractionation was conventional ( 2 Gy per daily fraction). With fractionated stereotactic radiation therapy and linac-based stereotactic radiosurgery the $\mathrm{NTD}_{\text {cumulative }}$ was even higher at $>105 \mathrm{~Gy}$ and $135 \mathrm{~Gy}$, respectively. Interestingly there was no difference in $\mathrm{NTD}_{\text {cumulative }}$ when latency period between radiation and re-irradiation was taken into account (up to 50 months). In summary, this study indicates that much higher doses to normal brain are possible with repeated irradiation treatments before necrosis becomes a serious risk.

\section{Case studies of re-irradiation of radiation-induced gliomas}

The following two case studies were taken from patients treated and followed long-term at the University of California San Francisco's Department of Radiation Oncology.

Case \#1: 14 year-old male with GBM 10 years after cranial irradiation-The patient was originally diagnosed with pre-B cell acute lymphoblastic leukemia at age 4 and was treated according to Children's Oncology Group (COG) protocol 1961 with 18 Gy of prophylactic cranial irradiation. Following treatment the patient remained clinically without evidence of disease until 14 years of age when he presented with severe headache and emesis for four days. Imaging (Figure 1A) revealed a $4 \mathrm{~cm}$ rim-enhancing mass in the patient's right frontal lobe concerning for high-grade glioma. The patient underwent neurosurgical intervention and a gross total resection was achieved with a final pathology of WHO grade IV astrocytoma. Since the patient had been treated previously with cranial irradiation, his case was presented at a multi-disciplinary neuro-oncology tumor board.

The decision was made to treat his disease with concurrent temozolomide and radiation. The radiation plan was a 3-dimentional conformal one using six beams delivering a total dose of 54 Gy (1.8Gy per daily fraction; 30 fractions total) prescribed to the $93 \%$ isodose line. The patient tolerated treatment very well and continued to attend school full-time. The patient was additionally enrolled in ACNS 0423 on which he received nine additional months of adjuvant temozolomide and lomustine (CCNU). Unfortunately, seven months after treatment for his initial GBM he recurred with multifocal disease in the cerebellum (Figure 1B). He was therefore re-treated to a dose of $36 \mathrm{~Gy}$ ( $3 \mathrm{~Gy}$ per daily fraction; 12 fractions total) prescribed to the $93 \%$ isodose line delivered concurrently with the anti-VEGF drug bevacizumab. The patient remains alive ( 14 months follow-up) at the time of this writing.

\section{Case \#2: 31 year-old female with GBM 20 years after cranial radiation and} intrathecal chemotherapy-The patient originally presented with acute myelogenous leukemia in 1981 at age 12 and her therapies included whole brain radiation of 18 Gy in addition to intrathecal chemotherapy. She did well until 31 years of age when she presented with a one week history of headache, nausea, vomiting, and decreased vision in her right eye. An MRI (Figure 2A) demonstrated a $6 \mathrm{~cm}$ solid, enhancing mass in the left occipital lobe. After gross total resection and final pathology of GBM, the patient was presented to our neuro-oncology tumor board for adjuvant treatment. Radiation treatment was provided at a total dose of $59.4 \mathrm{~Gy}$ (1.8 Gy per daily fraction; 33 fractions total) prescribed to the $93 \%$ isodose line using 3D conformal planning. Note that her treatment was administered prior to the publication of the landmark Stupp et al. trial [1].

Thirteen months after completion of her radiation, the patient presented with a new mass in her left frontal lobe (Figure 2B) in addition to new symptoms of ipsilateral headaches. The patient was re-treated with a fractionation scheme identical to her first treatment with the addition of concurrent temozolomide. One year later, the patient had recurrent disease 
adjacent to both of her resection cavities in the left occipital and frontal lobes (Figure 2C). Both of these lesions were treated using Gamma Knife stereotactic radiosurgery to a dose of $15 \mathrm{~Gy}$ at the 50\% isodose line. Unfortunately, the patient expired 9 months later though her overall survival from her initial GBM diagnosis was nearly three years.

\section{Expert Commentary}

Intracranial radiation in children can lead to a host of feared sequelae in addition to increasing the risk of radiation-induced gliomas. These sequelae include neuro-cognitive dysfunction [32] and hypothalamic-pituitary axis damage [33]. Despite its potential longterm side effects, radiation remains a key component of multimodality therapy for pediatric brain tumors. Some have suggested that the use of protons rather than x-rays may reduce the risk of secondary malignancies [34]. An important caveat is that in order to achieve this benefit protons must be delivered by active scanning rather than passive scattering. In the latter case, protons are scattered by a foil to cover the target; this can leads to production of neutrons that can increase the total-body dose up to 10-fold greater than IMRT. In adults, the risk of RIGs is far smaller than in children and the widespread proliferation of linear accelerators capable of conformal radiation should help mitigate increased risks of secondary malignancies.

It is clear that aggressive treatment for patients with RIGs is beneficial [3]. Since newer data suggest that the threshold for brain necrosis after re-irradiation may be higher than previously thought [29], the argument for irradiation of RIGs is correspondingly stronger. In addition to conventional therapy, molecular differences between RIGs and spontaneously occurring GBMs [9] support the enrollment of patients in small, multi-institutional clinical trials to exploit these differences [21-23]. As our two clinical cases illustrate, it is possible to re-treat patients for GBM recurrence with conformal radiation.

\section{Five-Year View}

In the next several years, improvements in radiation technology represent the most dramatic way by which the risk of RIGs may be mitigated. Since low-dose radiation on the edge of treatment fields is critically important to secondary cancer development [25], numerous studies have focused on radiation leakage from linear accelerators heads, scatter from multileaf collimators, and production of nuclear particles at high photon energies [35-37]. Methods suggested to mitigate this unnecessary radiation include shielding units for linear accelerator leakage, moving multi-leaf collimators to the field edge instead of fully retracting them, and using lower energy photon energies $(\sim 6 \mathrm{mV})$ to prevent neutron production. As these technologies are improved, the integral dose to patients given by photons should be dramatically reduced. Machines capable of delivering protons, long shown to have a theoretical advantage to photons due to their sharp dose fall-off, have rapidly proliferated world-wide. When using active scanning protons, also known as intensity modulated proton therapy (IMPT), it is possible to reduce the dose to healthy tissue in pediatric patients [38] compared to comparable photon devices.

\section{References}

1. Stupp R, Mason WP, van den Bent MJ, et al. Radiotherapy plus concomitant and adjuvant temozolomide for glioblastoma. N Engl J Med. 2005; 352(10):987-996. [PubMed: 15758009]

2. Ohgaki H, Kleihues P. Genetic pathways to primary and secondary glioblastoma. Am J Path. 2007; 170(5):1445-1453. [PubMed: 17456751]

3. Paulino AC, Mai WY, Chintagumpala M, Taher A, Teh BS. Radiation-induced malignant gliomas: is there a role for reirradiation. Int J Rad Biol Phys. 2008; 71(5):1381-1387. 
4. Neglia JP, Robison LL, Stovall M, et al. New primary neoplasms of the central nervous system in survivors of childhood cancer: a report from the childhood cancer survivor study. J Nat Can Inst. 2006; 98(21):1528-1537.

5. Sadetzki S, Chetrit A, Freedman L, Stovall M, Modan B, Novikov I. Long-term follow-up for brain tumor development after childhood exposure to ionizing radiation for tinea capitis. Rad Res. 2005; 163:424-432.

6. Karlsson P, Holmberg E, Lundell M, Mattsson A, Holm LE, Wallgren A. Intracranial tumors after exposure to ionizing radiation during infancy: a pooled analysis of two Swedish cohorts of 28,008 infants with skin hemangioma. Rad Res. 1998; 150:357-364.

7. Yonehara S, Brenner AV, Kishikawa M, et al. Clinical and epidemiologic characteristics of first primary tumors of the central nervous system and related organs among atomic bomb survivors in Hiroshima and Nagasaki, 1958-1995. Cancer. 2004; 101(7):1644-1654. [PubMed: 15378499]

8. Brat DJ, James CD, Jedlicka AE, et al. Molecular genetic alterations in radiation-induced astrocytomas. Am J Path. 1999; 154(5):1431-1438. [PubMed: 10329596]

9. Donson AM, Erwin NS, Kleinschmidt-DeMasters BK, Madden JR, Addo-Yobo SO, Foreman NK. Unique molecular characteristics of radiation-induced glioblastoma. J Neuropath Exp Neurol. 2007; 66(8):740-749. [PubMed: 17882018]

10. Cardis E, Vrijheid M, Blettner M, et al. The 15-country collaborative study of cancer risk among radiation workers in the nuclear industry: estimates of radiation-related cancer risks. Rad Res. 2007; 167:396-416.

11. Rahu M, Rahu K, Anuvinen A, et al. Cancer risk among Chernobyl cleanup workers from Estonia and Latvia, 1986-1998. Int J Can. 2006; 119:162-168.

12. Sodickson A, Baeyens PF, Andriole KP, et al. Recurrent CT, cumulative radiation exposure, and associated radiation-induced cancer risks from CT of adults. Radiology. 2009; 251(1):175-184. [PubMed: 19332852]

13. Cahan WG, Woodard HQ, Higinbotham NL, Stewart FW, Coley BL. Sarcoma arising in irradiated bone: report of 11 cases. 1948. Cancer. 1998; 82(1):6-34. [PubMed: 9428475]

14. Linet MS, Kim KP, Rajaraman P. Children's exposure to diagnostic medical radiation and cancer risk: epidemiologic and dosimetric considerations. Pediatr Radiol. 2009; 39(Suppl 1):S4-S26. [PubMed: 19083224]

15. Howe GR, Burch JD, Chiarelli AM, Risch HA, Choi BC. An exploratory case-control study of brain tumors in children. Can Res. 1989; 49:4349-4352.

16. McCredie M, Maisonneuve P, Boyle P. Perinatal and early postnatal risk factors for malignant brain tumors in New South Wales children. Int J Cancer. 1994; 56:11-15. [PubMed: 8262665]

17. Shu XO, Jin F, Linet MS, et al. Diagnostic x-ray and ultrasound exposure and risk in childhood cancer. Br J Cancer. 1994; 70(3):531-536. [PubMed: 8080742]

18. Schuz J, Kaletsch U, Kaatsch P, Meinert R, Michaelis J. Risk factors for pediatric tumors of the central nervous system: results from a German population-based case-control study. Med Pediatr Oncol. 2001; 36:274-282. [PubMed: 11452935]

19. Kuijten RR, Bunin GR, Nass CC, Meadows AT. Gestational and familial risk factors for childhood astrocytoma: results of a case-control study. Can Res. 1990; 50:2608-2612.

20. Bunin GR, Buckley JD, Boesel CP, Rorke LB, Meadows AT. Risk factors for astrocytic glioma and primitive neuroectodermal tumor of the brain in young children: a report from the Children's Cancer Group. Cancer Epi Bio Prev. 1994; 3:197-204.

21. Contessa JN, Bhojani MS, Freeze HH, Rehemtulla A, Lawrence TS. Inhibition of N-linked glycosylation disrupts receptor tyrosine kinase signaling in tumor cells. Can Res. 2008; 68(10): 3803-3809.

22. Wester MD, Wasteson A, Lindstrom A. Targeting malignant glioma cells in vitro using plateletderived growth factor AA-based conjugates. J Drug Target. 2009; 17(4):268-277. [PubMed: 19255898]

23. Ferletta M, Uhrbom L, Olofsson T, Ponten F, Westermark B. Sox10 has a broad expression pattern in gliomas and enhances platelet-derived growth factor-B-induced gliomagenesis. Mol Cancer Res. 2007; 5(9):891-897. [PubMed: 17855658] 
24. Nieder C, Astner ST, Mehta MP, Grosu AL, Molls M. Improvement, clinical course, and quality of life after palliative radiotherapy for recurrent glioblastoma. Am J Clin Oncol. 2008; 31(3):300305. [PubMed: 18525311]

25. Diallo I, Haddy N, Adjadj E, et al. Frequency distribution of second solid cancer locations in relation to the irradiated volume among 115 patients treated for childhood cancer. Int J Rad Biol Phys. 2009; 74(3):876-883.

26. Klein EE, Maserang B, Woord R, Mansur D. Peripheral doses from pediatric IMRT. Med Phys. 2006; 33(7):2525-2531. [PubMed: 16898456]

27. Mansur DB, Klein EE, Maserang BP. Measured peripheral dose in pediatric radiation therapy: a comparison of intensity-modulated and conformal techniques. Radiother Oncol. 2007; 82:179184. [PubMed: 17257700]

28. Emami B, Lyman J, Brown A, et al. Tolerance of normal tissue to therapeutic irradiation. Int J Radiat Oncol Biol Phys. 1991; 21(1):109-22. [PubMed: 2032882]

29. Mayer R, Sminia P. Reirradiation tolerance of the human brain. Int J Radiat Oncol Biol Phys. 2008; 70(5):1350-1360. [PubMed: 18037587]

30. Brenner DJ, Curtis RE, Hall EJ, Ron E. Second malignancies in prostate carcinoma patients after radiotherapy compared with surgery. Cancer. 2000; 88(2):398-406. [PubMed: 10640974]

31. Boice JD Jr, Engholm G, Kleinerman RA, et al. Radiation dose and second cancer risk in patients treated for cancer of the cervix. Radiat Res. 1988; 116(1):3-55. [PubMed: 3186929]

32. Askins MA, Moore BD 3rd. Preventing neurocognitive late effects in childhood cancer survivors. J Child Neurol. 2008; 23(10):1160-71. [PubMed: 18952582]

33. Karachaliou F, Simatos G, Batika P, Michalacos S, Kaldrymides P. Endocrine consequences of childhood malignancies. J BUON. 2009; 14(1):27-32. [PubMed: 19373943]

34. Hall EJ. Intensity-modulated radiation therapy, protons, and the risk of second cancers. Int J Radiat Biol Phys. 2006; 65(1):1-7.

35. Stern RL. Peripheral dose from a linear accelerator equipped with multileaf collimation. Med Phys. 1999; 26(4):559-63. [PubMed: 10227359]

36. Robinson D, Scrimger JW, Field GC, Fallone BG. Shielding considerations for tomotherapy. Med Phys. 2000; 27(10):2380-4. [PubMed: 11099208]

37. Chibani O, Ma CM. Photonuclear dose calculations for high-energy photon beams from Siemens and Varian linacs. Med Phys. 2003; 30(8):1990-2000. [PubMed: 12945965]

38. Fogliata A, Yartsev S, Nicolini G, et al. On the performances of Intensity Modulated Protons, RapidArc and Helical Tomotherapy for selected paediatric cases. Radiat Oncol. 2009; 4:2. [PubMed: 19144155]

101. CBTRUS 2009. CBTRUS Statistical Report: Primary Brain and Central Nervous System Tumors Diagnosed in the United States in 2004-2005. Central Brain Tumor Registry of the United States; 2009. Available at: http://www.cbtrus.org/reports/2009-NPCR-04-05/CBTRUS-

NPCR2004-2005-Report-.pdf [May 15, 2009]

102. Phase II Pilot Study of Adjuvant Temozolomide Concurrently With Postoperative Radiotherapy and Then Alone As Maintenance Therapy in Children With Newly Diagnosed Anaplastic Astrocytoma, Glioblastoma Multiforme, Gliosarcoma, or Diffuse Intrinsic Pontine Glioma. National Cancer Institute; Available at: http://www.cancer.gov/clinicaltrials/COG-ACNS0126 [May 15, 2009] 


\section{Key Issues}

- Radiation-induced gliomas (RIGs) are secondary malignancies that can occur years after intracranial radiation exposure with either therapeutic or diagnostic intent.

- Children (especially those under 5 years of age) are at greater risk for RIGs than adults.

- The incidence of RIGs appears to be volume-dependent and tissues that receive low-doses of radiation are particularly susceptible.

- Microarray analysis suggests that RIGs are genetically distinct from spontaneously occurring glioblastomas and, in fact, may have more in common with low-grade gliomas.

- Without re-irradiation, patients with RIGs have a significantly poorer prognosis. Data from re-irradiated brains indicate that this organ may be more radioresistant than previously thought.

- Current and future technologies to reduce the risk of RIGs include linear accelerator shielding and use of active scanning protons. 

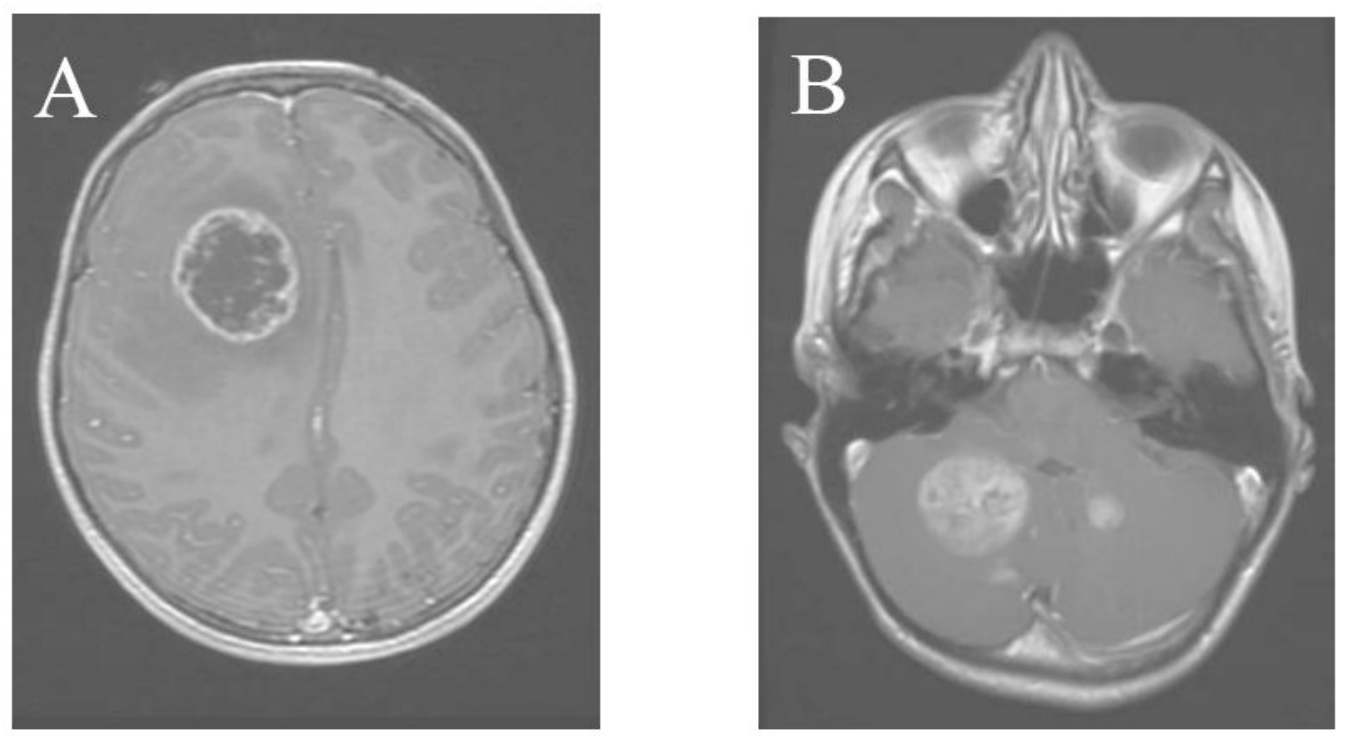

Figure 1. Post-gadolinium T1-weighted MRI of 14 year-old male approximately 10 years after prophylactic cranial irradiation for pre-B cell acute lymphoblastic leukemia

(A) Patient has a $4 \mathrm{~cm}$ cystic mass in the right frontal lobe with surrounding edema. Pathology revealed WHO grade IV astrocytoma (GBM). (B) 7 months after treatment of initial disease the patient presented with recurrent multi-focal disease in the cerebellum. The patient is currently without clinical evidence of disease at 14 months of follow up. 

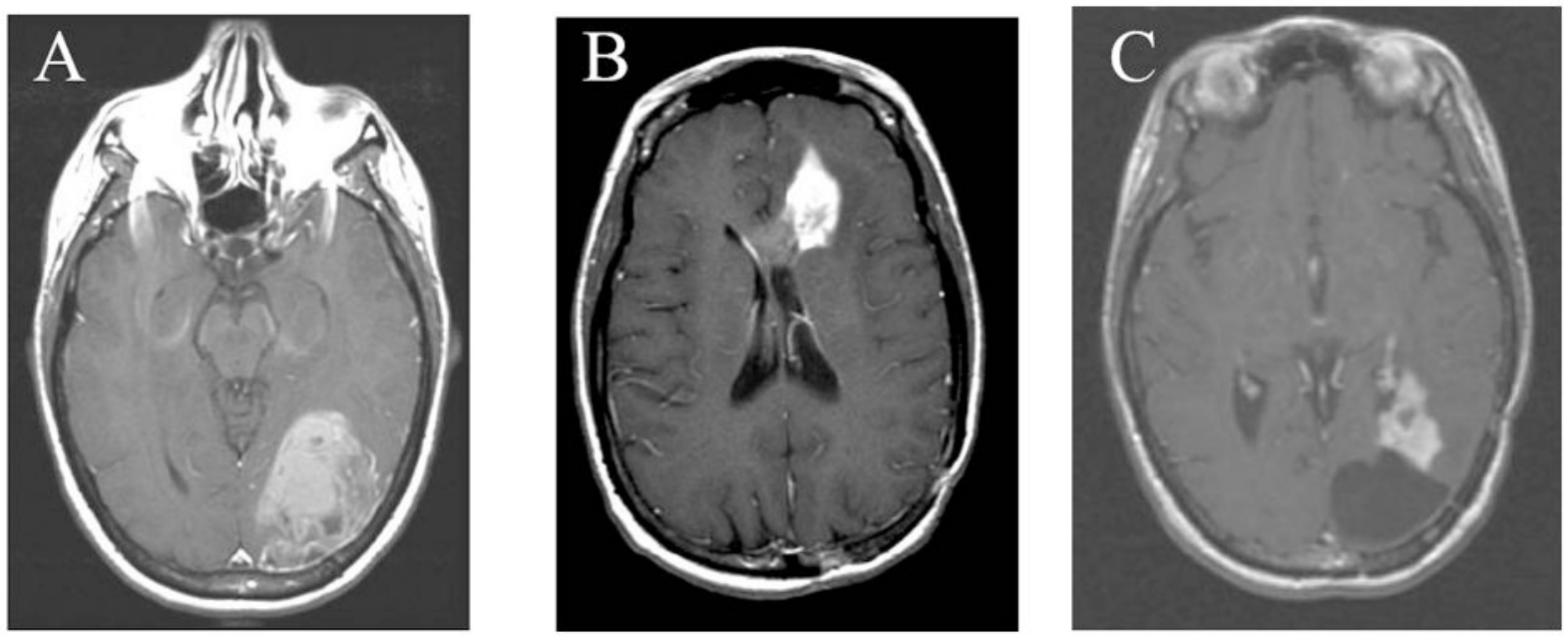

Figure 2. Post-gadolinium T1-weighted MRI of 31 year-old female approximately 20 years after whole-brain radiation and intrathecal methotrexate for acute myelogenous leukemia Pt had a $6 \mathrm{~cm}$ enhancing mass in the left occipital lobe. Pathology revealed WHO grade IV astrocytoma (GBM). (B) 14 months after her initial GBM treatment, the patient presented with a recurrence in her left frontal lobe. (C) 13 months after her GBM the patient had recurrence adjacent to her two resection cavities in the left occipital (shown) and left frontal lobes. The patient passed away 34 months after her GBM diagnosis. 
Table 1

Risks of secondary glioma after irradiation in pediatric population

\begin{tabular}{ccccc}
\hline Indication for Radiation & Dose Range & ERR & Tumor & Ref. \\
\hline Cancer, all types & $0-45+\mathrm{Gy}$ & $0.33 / \mathrm{Gy}^{*}$ & Glioma & {$[4]$} \\
Tinea Capitis & $1.4 \mathrm{~Gy}$ (median) & $1.98 / \mathrm{Gy}^{*}$ & Malignant BT & {$[5]$} \\
Skin Hemangioma & $0.02 \mathrm{~Gy}$ (median) & $2.7 / \mathrm{Gy}^{*}$ & Intracranial Tumors & {$[6]$} \\
Skull Radiograph & $0.00015 \mathrm{~Sv}$ & $6.7^{*}$ & Intracranial Tumors & {$[15]$} \\
Skull Radiograph & $0.00015 \mathrm{~Sv}$ & 2.3 & Intracranial Tumors & {$[16]$} \\
All Diagnostic X-rays/Ultrasounds & Variable (but low) & 1.5 & Childhood Tumors & {$[17]$} \\
All Diagnostic X-rays/Ultrasounds & Variable (but low) & 0.7 & Intracranial Tumors & {$[18]$} \\
Head or Neck X-ray & Variable (but low) & 1 & Astrocytoma & {$[19]$} \\
Head, Neck or Dental X-ray & Variable (but low) & 1.2 & Astrocytoma & {$[20]$}
\end{tabular}

Abbreviations: $\mathrm{ERR}=$ effective relative dose,

* statistically significant, $\mathrm{BT}=$ brain tumor 